\title{
THE INFLUENCE OF TURBULENCE MODEL AND TWO AND THREE-DIMENSIONAL DOMAIN SELECTION ON THE SIMULATED PERFORMANCE CHARACTERISTICS OF VERTICAL AXIS TIDAL TURBINES
}

\author{
Philip Marsh ${ }^{1 a}$, Dev Ranmuthugala², Irene Penesis ${ }^{1}$ and Giles Thomas ${ }^{3}$
}

${ }^{1}$ National Centre for Maritime Engineering and Hydrodynamics, Australian Maritime College, University of Tasmania. Locked Bag 1395, Launceston Tasmania 7250, Australia

${ }^{2}$ National Centre for Ports and Shipping, Australian Maritime College, University of Tasmania. Locked Bag 1397, Launceston Tasmania 7250, Australia

${ }^{3}$ UCL Mechanical Engineering, University College London, Torrington Place, London WC1E 7JE

aCorresponding author: marshp@amc.edu.au. Locked Bag 1395, Launceston Tasmania 7250, Australia. Phone: (+61) 401639059

\begin{abstract}
The influence of Computational Fluid Dynamics (CFD) modeling techniques on the accuracy of vertical axis turbine power output predictions was investigated. Using Two-Dimensional (2D) and Three-Dimensional (3D) models, as well as the Baseline-Reynolds Stress Models (BSL-RSM) model and the $k-\omega$ Shear Stress Transport ( $k-\omega S S T)$ model in its fully turbulent and laminar-to-turbulent formulation, differences in power output modeling accuracy were evaluated against experimental results from literature. The highest correlation with experimental power output was found using a 3D domain model that fully resolved the boundary layer combined with the $k-\omega$ SST laminar-to-turbulent model. The turbulent 3D fully resolved boundary layer $k-\omega$ SST model also accurately predicted power output for most rotational rates, at a significantly reduced computational cost when compared to its laminar-to-turbulent formulation. The 3D fully resolved BSL-RSM model and 3D wall function boundary layer $k-\omega S S T$ model were found to poorly simulate power output. Poor output predictions were also obtained using 2D domain $k-\omega$ SST models, as they were unable to account for blade tip and strut effects. The authors suggest that 3D domain fully turbulent $k-\omega$ SST models with fully resolved boundary layer modeling are used for predicting turbine power output given their accuracy and computational efficiency.
\end{abstract}

Keywords: Vertical Axis Turbine, Computational Fluid Dynamics, Turbulence Model, Computational Domain, Laminar-to-Turbulent Transition

\section{Highlights:}

- 2D CFD models cannot capture blade tip and strut effects resulting in poor power output simulation accuracy

- 3D CFD models can now accurately capture turbine power output without excessive computation requirements

- The $k-\omega$ SST turbulence models provide the closest agreement with experimental results

- Transition flow modeling is computationally demanding and only increases simulation accuracy at high rotational rates when compared to fully turbulent models

- BSL-RSM and $k-\omega$ SST Wall Function models poorly simulate power output 


\section{INTRODUCTION}

The potential of using ocean energy to generate renewable energy has led to increased interest in vertical axis turbines, as they are a viable option to transform the ocean's tidal and current kinetic energy into electricity [1]. Vertical axis fixed pitch cross flow turbine designs exhibit two key advantages over competing ocean energy device designs: they are insensitive to inflow direction and all electrical components can be mounted above the water surface [2]. When combined, these factors enable simple and robust turbine designs for ocean installations. However, little is known of their complex hydrodynamic properties, as previous research and development has concentrated on horizontal axis designs as commonly utilised in the wind energy industry [3]. If ocean turbines are to be successfully deployed at commercial scales detailed understanding of their operational characteristics is essential; this can be obtained through numerical simulations such as Computational Fluid Dynamics (CFD) or by Experimental Fluid Dynamics (EFD).

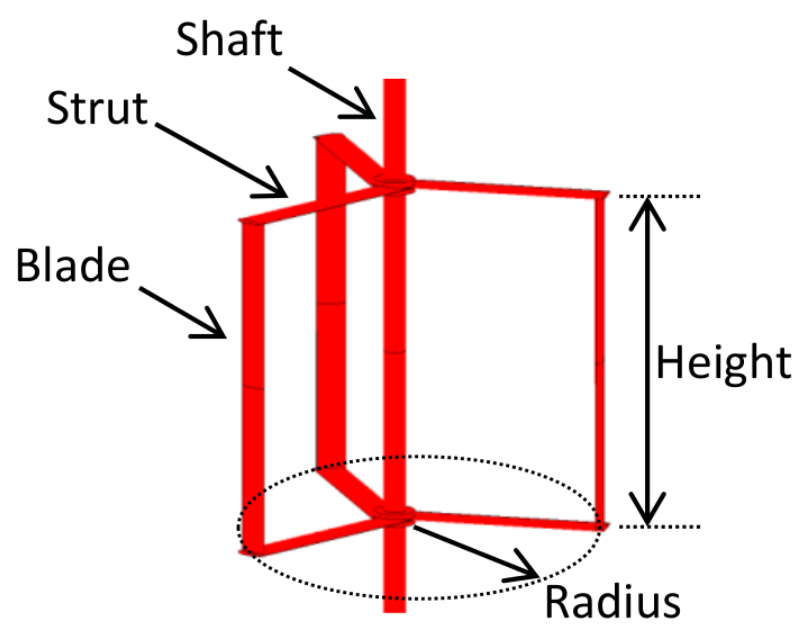

Figure 1: Vertical Axis Turbine showing definitions of key geometrical features

Vertical axis turbines are commonly studied using CFD models as they allow for turbine performance investigations without the expense of EFD. The majority of CFD simulations are performed using Two-Dimensional (2D) models that are generated using thin slices of the turbine geometry in the horizontal plane, with no blade tips, struts, or shafts modeled. These 2D models are often used due to their significantly reduced computational requirements when compared to Three-Dimensional (3D) models. However, these 2D approaches often significantly over predict maximum power output [4-7] due to the highly 3D nature of turbine hydrodynamic flow due to blade and strut joint and blade tip losses [8]. Numerical simulations are also commonly performed using fully turbulent models [4-8], again due to their computational efficiency. However, the influence of laminar-to-turbulent flow transition on power output predictions is unknown, and may be significant as turbines can operate in low Reynolds number dominated flows [8]. For turbulence modeling the $k-\omega$ Shear Stress Transport ( $k-\omega$ SST) model is prevalent [2,4,8-14], due to its ability to model both free stream and wall bounded flows accurately. However, studies of the influence of alternative turbulence modeling schemes on the accuracy of power output predictions are limited. Combined, the influence of these modeling factors on power output predictions is unknown and was the key driver for this research.

Numerical simulations were performed on two vertical axis turbines using the ANSYS CFX software package to evaluate the influence of turbulence model and $2 \mathrm{D}$ and $3 \mathrm{D}$ domain modeling techniques on the predictive capacity of Unsteady Reynolds Averaged Navier-Stokes (URANS) based simulation models [15]. These turbine models were geometrically identical to EFD models 
from literature [16] to allow for verification and validation of all power output simulations. The 2D and 3D CFD models developed used the $k-\omega$ SST and Baseline-Reynolds Stress Models (BSL-RSM) turbulence models with differing wall modeling approaches to appraise any wall modeling influences on simulation accuracy. Additionally, flow transition modeling was performed using the $k-\omega$ SST Gamma-Theta Transition ( $k-\omega$ SST Transition) model to establish the influence of transition modeling on power output simulation accuracy.

\section{NUMERICAL SIMULATION}

Numerical 2D and 3D CFD simulations were performed using the ANSYS CFX software package [15], which solves the transient URANS equations using an element-based finite volume approach. Simulations of two geometrically different turbines were performed to evaluate the ability of the various turbulence and $2 \mathrm{D}$ and $3 \mathrm{D}$ domain models to accurately simulate power output when compared to EFD from literature [16].

\subsection{Turbine Geometry and Experimental Fluid Dynamics (EFD) Models}

Two straight-bladed vertical axis turbines models were developed as shown in Figure 2, labeled Turbines $A$ and $B$ respectively. These turbine designs were geometrically equivalent to EFD models from literature [16] to allow validation of the numerical simulation approaches. These models shared the same blade section, span, and number of blades as detailed in Table 1. However, the turbines differed in strut section and location, as Turbine A had NACA0012 strut sections located at the blade ends, while Turbine $B$ had shaped bar struts located at the blade quarter span, both shown in Figure 1. The EFD testing from literature was conducted at full scale, with chord Reynolds numbers ranging from approximately 50,000 to 500,000 [16].

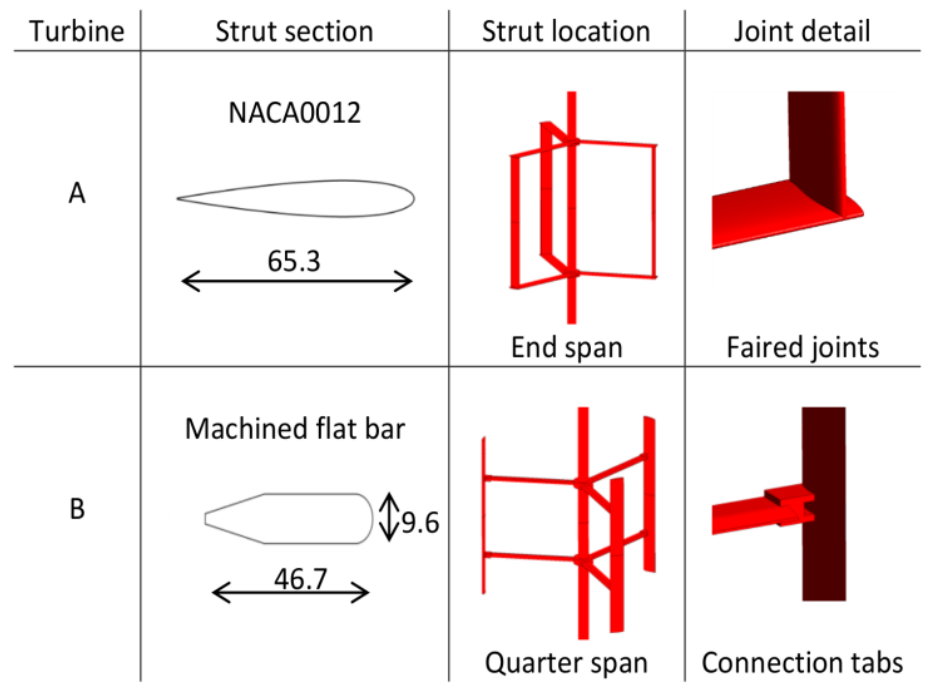

Figure 2: Turbine A and B strut section and location detail. Dimensions in mm [16]

Table 1: Shared Geometry of Turbines A and B [16]

\begin{tabular}{cc}
\hline Geometry & Dimensions \\
\hline Number of blades & 3 \\
Number of struts & 2 per blade \\
Blade section & NACA 634021 \\
Blade chord & $0.065 \mathrm{~m}$ \\
Diameter (d) & $0.915 \mathrm{~m}$ \\
Turbine span (s) & $0.686 \mathrm{~m}$ \\
\hline
\end{tabular}




\subsection{Key Performance Parameters}

To evaluate turbine performance, the turbine power output is evaluated as the non-dimensionalised power coefficient $C_{p}$ where,

$$
C_{p}=\lambda C_{m}
$$

where the tip speed ratio $\lambda$ is defined as,

$$
\lambda=r \omega / V
$$

and $\omega$ is the turbine rotational rate, $r$ is the turbine radius, $V$ is the inflow velocity, and the turbine torque $C_{m}$ is determined as,

$$
C_{m}=\frac{\text { Torque }}{0.5 \rho V^{2} S r}
$$

where $\rho$ is the water density (set to $997 \mathrm{~kg} / \mathrm{m}^{3}$ for all simulations), $S$ is the turbine frontal area, and the Torque generated by the turbine was taken from the respective CFD or EFD results.

\subsection{Turbulence and Boundary Layer Modeling}

The influence of turbulence model selection on power output predictions was examined using three turbulence models; the $k-\omega$ SST, $k-\omega$ SST Transition and BSL-RSM models. Differing boundary layer modeling techniques were also introduced to evaluate the influence of boundary layer modeling techniques on simulation accuracy.

The influence of flow transition was modeled using the Gamma-Theta formulation of the $k-\omega$ SST transition model [15]. The $k-\omega$ SST Transition model uses two additional transport equations when compared to the fully turbulent $k-\omega$ SST model to capture transitional effects: the intermittency equation, $\gamma$, and the transition onset momentum thickness Reynolds number, Re- $\theta$. [15]. The $k-\omega$ SST transition model does not model the physical fluid processes, but rather applies empirical correlations to the underlying $k-\omega$ SST model [15]. For this study no modification of correlation coefficients was performed, as post priori modification of these coefficients would reduce the model's utility for design investigations where performance data was unavailable. To ensure the accurate simulation of flow near all turbine surfaces, the boundary layers were fully resolved as recommended [15].

The $k-\omega$ SST turbulence model was also used in its fully turbulent formulation as it is commonly used to simulate turbine performance [2,4,8-14] due to its ability to model both the boundary layer and the free stream regions. Research has shown that it can accurately predict flow separation and adverse pressure gradients as transport effects are included into the formulation of the eddy-viscosity equations [15,17]. To evaluate the influence of boundary layer modeling two 3D $k-\omega$ SST meshes were developed, with flow modeling near the turbine surfaces performed either by using prescribed wall functions based on log-wall laws or by fully resolving the boundary layer flow [15]. This resulted in the two 3D $k-\omega$ SST meshes using differing inflation layer densities as shown in Figure 3, which are referred to as the 3D $k-\omega$ SST Wall Function or 3D $k-\omega$ SST models respectively. The $k-\omega S S T$ turbulence model was also used on a $2 \mathrm{D}$ mesh, referred to as the $2 \mathrm{D}$ $k-\omega$ SST model. Total boundary layer thickness was estimated as $0.37 c / R e^{\frac{1}{5}}$ with the blade chord $c$ used to determine Reynolds number, $R e$ [18]. This estimated boundary layer thickness was doubled to ensure that the boundary layer was contained within the prescribed inflation layer region. Inflation layer mesh growth rates were limited to 1.2 as recommended [15]. Boundary layer mesh density 
independence was also evaluated by examining the influence on power output of the average height of the first cell from the turbine walls, known as the non-dimensional variable $y+[15]$.

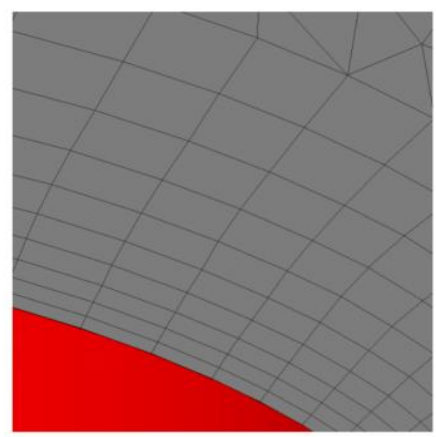

(a)

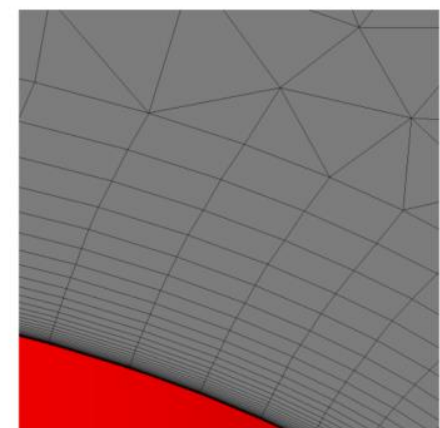

(b)

Figure 3: Blade surface inflation layer mesh density for: (a) $k-\omega$ SST Wall Function and (b) fully resolved 3D $k-\omega$ SST models showing differences in mesh density with 10 and 30 layers respectively

Along with the two $k-\omega$ SST-based turbulence models, power output simulations were performed using the BSM-RSM model, which closes the URANS equations by solving six transport equations for the Reynolds stresses, as well as an additional equation for dissipation rate [15]. Due to these additional transport equations BSL-RSM is suggested to be better at modeling complex flows with high levels of streamline curvature, fluid rotation, and rotating reference frames [15], as occurs during vertical axis turbine operations. To ensure the accurate simulation of flow near all turbine surfaces, the boundary layers were fully resolved.

The influence of numerical discretization scheme on $C_{p}$ prediction accuracy was investigated by performing simulations using low and high order numerical schemes, with results compared to EFD from literature for Turbine A [16]. The high order scheme used a bounded second order upwind-biased advection and an unbounded second order backwards Euler transient scheme, whereas the lower order scheme used a first order upwind advection and first order backwards Euler transient scheme [15].

For all simulations the fluid was modeled as incompressible as all flow velocities were significantly less than Mach 0.3 . An inlet turbulence setting of $5 \%$ was applied as no measurements of turbulence intensity were provided from the EFD testing from literature [16]. Convergence was deemed achieved when solution residuals reduced to below $10^{-4}$ and reduced by more than three orders of magnitude. Additionally convergence was confirmed by ensuring that the final $C_{p}$ determined was within $5 \%$ of the previous rotations results, required due to the periodic nature of $C_{p}$. An example of $C_{p}$ convergence for Turbine $\mathrm{A}$ is shown in Figure 4, where $C_{p}$ values converged after approximately 3600 time steps, corresponding to 9 rotations. To reduce overall simulation times all simulations were started using previous simulation results if available, reducing the initialization process and thus the overall computational requirements. The oscillation of $C_{p}$ shown in Figure 4 results from variations in blade instantaneous torque as each blade generates peaks in torque as they rotate through the region upstream of the turbine shaft [16]. 


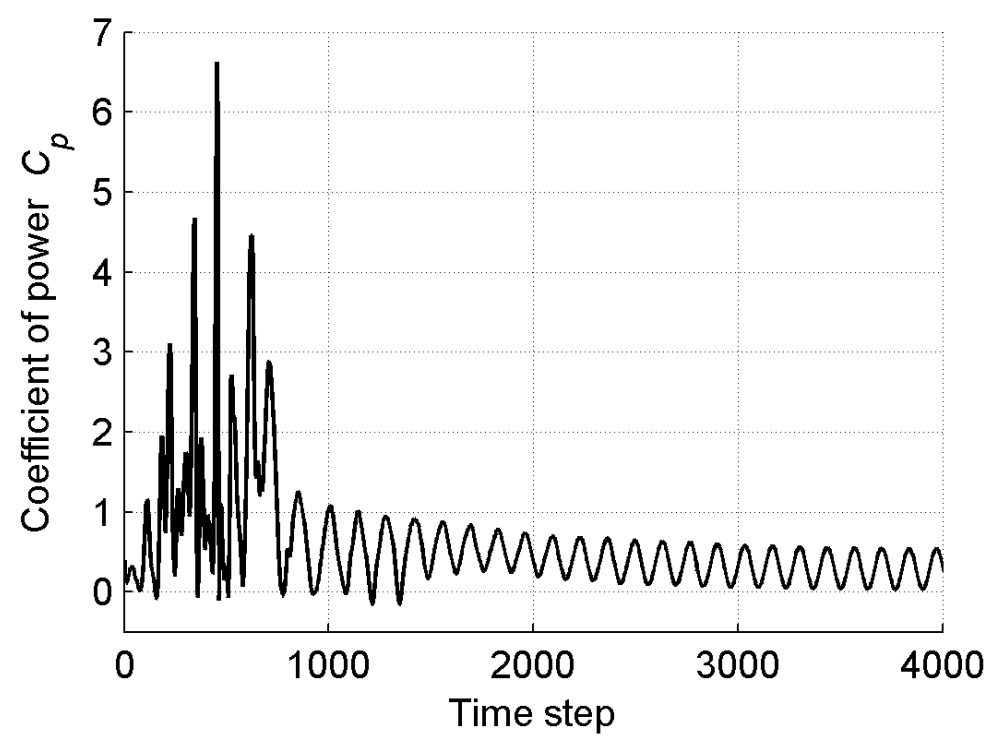

Figure 4: Example of $C_{p}$ convergence for Turbine $A$ at $\lambda=2.75$ at an inflow velocity of $1.5 \mathrm{~ms}^{-1}$

\subsection{Computational Fluid Dynamics (CFD) Models}

To determine the influence of domain selection on simulation accuracy, both $2 \mathrm{D}$ and $3 \mathrm{D}$ domains were generated. All turbine models were meshed using ANSYS CFX 13.0 [15] using unstructured tetrahedral elements, an example of which is shown in Figure 5 for the 3D $k-\omega$ SST model. The 3D CFD models included all 3D geometrical features including all blades, struts, hubs, and shaft, with the $k-\omega$ SST and BSL-RSM models using the same mesh. The 2D models only included the blades and shaft due to the geometrical layout of vertical axis turbines. Turbine rotation was simulated by enclosing the turbine in an inner domain as shown in Figure 3. This domain was rotated using the CFX transient rotor-stator model [15], with a General Grid Interface (GGl) used to interpolate flow values across the interface due to non-conformal mesh. To minimise any errors in the intersection algorithm, the GGI was placed at 1.5 times the turbine diameter measured from the rotational axis. Mesh density was also increased on this interface as shown in Figure 3 to further minimise any errors across the GGI interface. For all simulations the inner domain was rotated at the desired rotational rate corresponding to CFD or EFD testing rates. Mesh density was varied according to expected flow curvature rates, resulting in increased density in regions near the blades, struts, hubs, shaft, and wake. Mesh density was reduced away from the turbine surfaces such as near the computational domain boundaries to minimise computational effort.

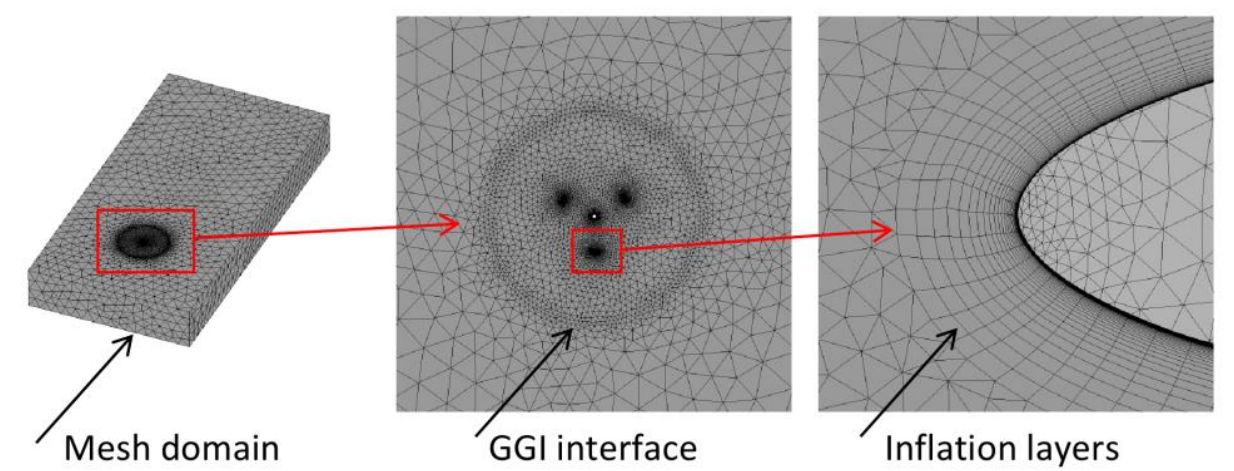

Figure 5: Mesh domain for 3D model showing overall mesh domain, GGI interface, and inflation layer detail on the blade surface for the 3D $k-\omega$ SST Turbine A model 
The 3D computational domains were generated to isolate the turbine from any boundary effects; with all boundary conditions outlined in Figure 6 and Table 2, as determined by the systematic domain size independence studies outlined in Section 2.5. Both Turbines A and B were assumed to operate at sufficient depths to ignore free surface effects, allowing the use of symmetry to reduce domain size $[2,8,13,14]$. Full and half domains were generated, with the half domain split along the horizontal mid plane. To ensure that this use of a half domain did not influence the accuracy of the power output predictions, simulations using a full 3D domain, also shown in Figure 6, were performed for comparison.

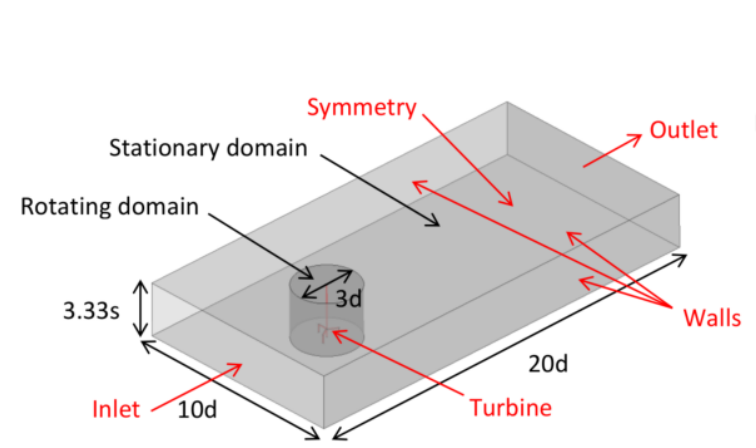

(a)

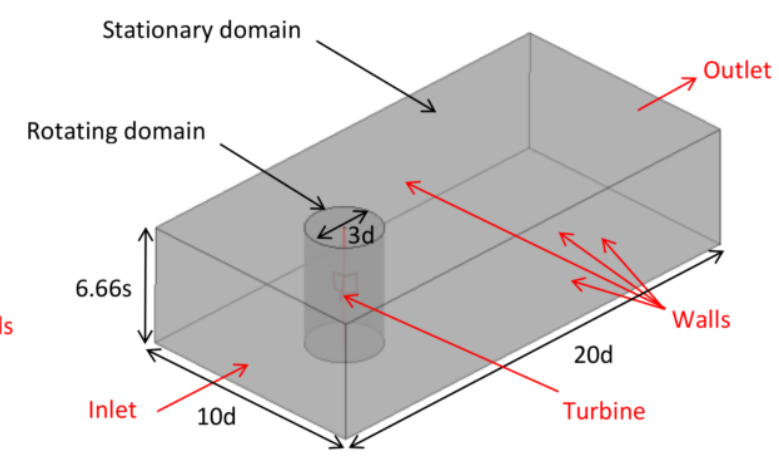

(b)

Figure 6: Domain nomenclature and sizing for the (a) half, and (b) full 3D CFD models. Dimensions in turbine diameter $\mathrm{d}$ and span $\mathrm{s}$

Table 2: Domain Boundary Conditions for all CFD Models

\begin{tabular}{cc}
\hline Wall & Boundary condition \\
\hline Inlet & Uniform flow perpendicular to inlet wall: $1.5 \mathrm{~ms}^{-1}$ \\
Outlet & Relative Pressure: $0 \mathrm{~Pa}$ \\
Walls & Free slip walls \\
Turbine & No slip walls \\
Symmetry & Symmetry walls \\
\hline
\end{tabular}

The 2D CFD model is shown in Figure 7, with the boundary details outlined in Table 2. In comparison to the 3D CFD model, shown in Figure 6, the 2D CFD model consisted only of the blade sections and shaft. This resulted in the same model being used for Turbines $A$ and $B$, as the models differed only in strut location and section. The computation domain was constructed by cutting a $0.01 \mathrm{~m}$ slice from the 3D model shown in Figure 6, as ANSYS CFX cannot natively model in 2D, with the domain thickness represented by a thin layer of tetrahedral cells. This model was referred to as the 2D $k-\omega$ SST model, as simulations were only performed on the 2D domain using the $k-\omega$ SST turbulence model.

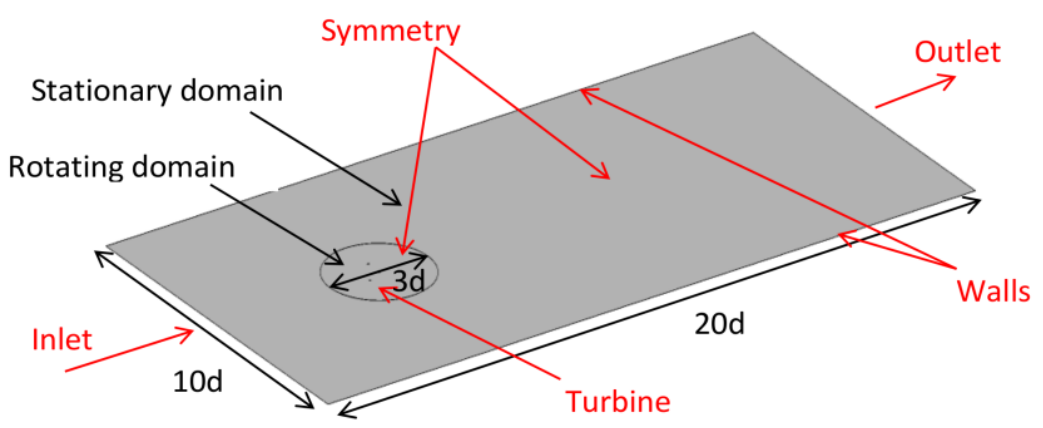

Figure 7: Domain boundary nomenclature and sizing for 2D domain. Total 2D domain thickness of $0.01 \mathrm{~m}$. Dimensions in turbine diameter $\mathrm{d}$. 


\subsection{Mesh Independence Studies}

Systematic independence studies were performed to ensure domain size, domain boundary, mesh density, boundary layer modeling, and time step independence for both turbine designs. Independence was evaluated by investigating the impact on power output of increases in these factors until variations between each successive refinement reduced to less than $5 \%$. Examples of the independence studies are only presented for Turbine A here for brevity, but were performed for both turbines with similar results found. The influence of numerical discretisation scheme on $C_{p}$ was also evaluated.

Figure 8 illustrates mesh element count independence for Turbine A, which was achieved at $0.16,9.1,17.2,17.2$, and 27.2 million elements for the 2D $k-\omega$ SST, 3D $k-\omega$ SST Wall Function, 3D BSL-RSM, 3D $k-\omega$ SST, and 3D $k-\omega$ SST Transition models respectively, with Turbine B CFD models exhibiting similar mesh element counts. The 2D model demonstrated low mesh element count independence at 0.16 million elements as a result of the significant reduction in domain size as seen when comparing Figures 6 and 7. The lower mesh count for the 3D $k-\omega$ SST Wall Function model was due to the significantly reduced inflation layer density shown in Figure 3 when compared to the fully resolved models. Compared to the 3D BSL-RSM and 3D $k-\omega$ SST models, the 3D $k-\omega$ SST Transition model required higher mesh density in the chord wise direction as recommended for transition region prediction [15].
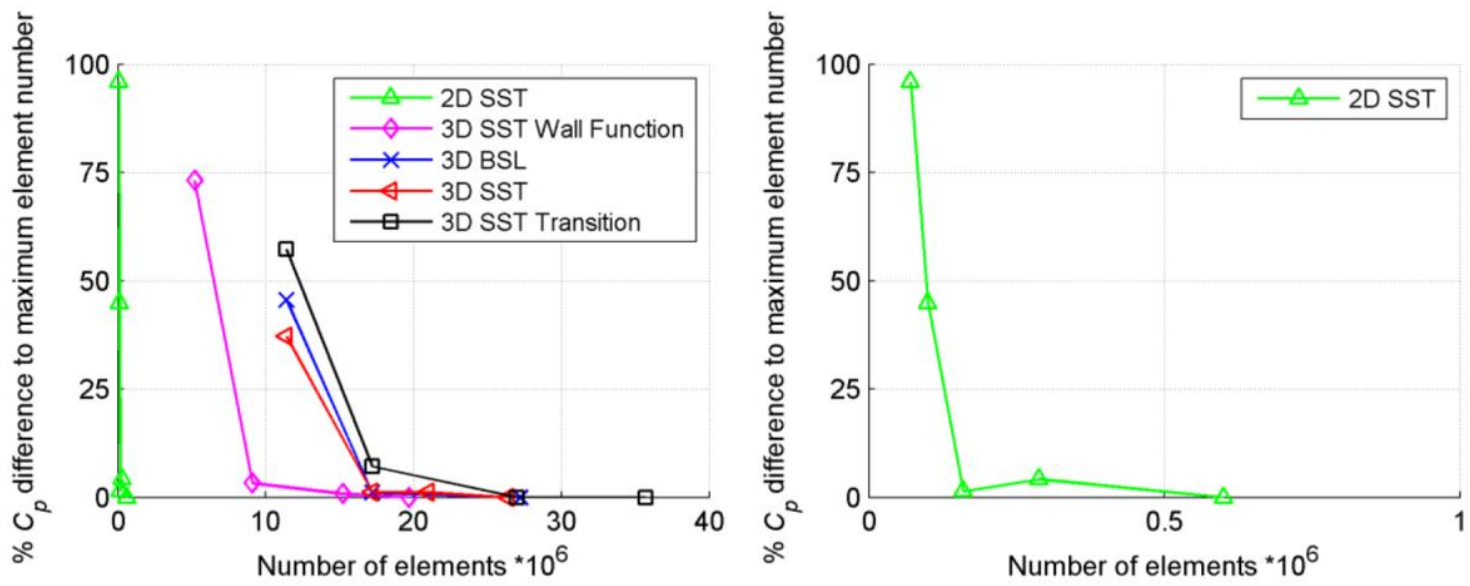

Figure 8: Mesh element count independence for Turbine A CFD models at $\lambda=2.75$ and an inflow velocity of $1.5 \mathrm{~ms}^{-1}$. Results for 2D SST model shown on right for clarity

Simulations of 2D and 3D CFD models revealed significant differences in temporal independence as shown in Figure 9 for Turbine A. Temporal independence was demonstrated at $0.9^{\circ}$ rotation per time step for all models except the 3D $k-\omega$ SST Wall Function model, which demonstrated temporal independence at $3.6^{\circ}$ of rotation per time step. The increase in time step size for the 3D $k-\omega$ SST Wall Function model was caused by the reduction in boundary layer density. The 2D $k-\omega$ SST results were similar to the 3D $k-\omega$ SST as they used the same boundary layer mesh density. Small fluctuations in power output were noted for some simulations as time step reduced, as shown for the 3D $k-\omega$ SST model between $0.9^{\circ}$ and $0.225^{\circ}$. This is ascribed to small differences in wake and dynamic stall modeling between the successive refinements. However as these fluctuations were lower than the independence criteria they were ignored, allowing the use of higher time steps to maximise computational efficiency. 


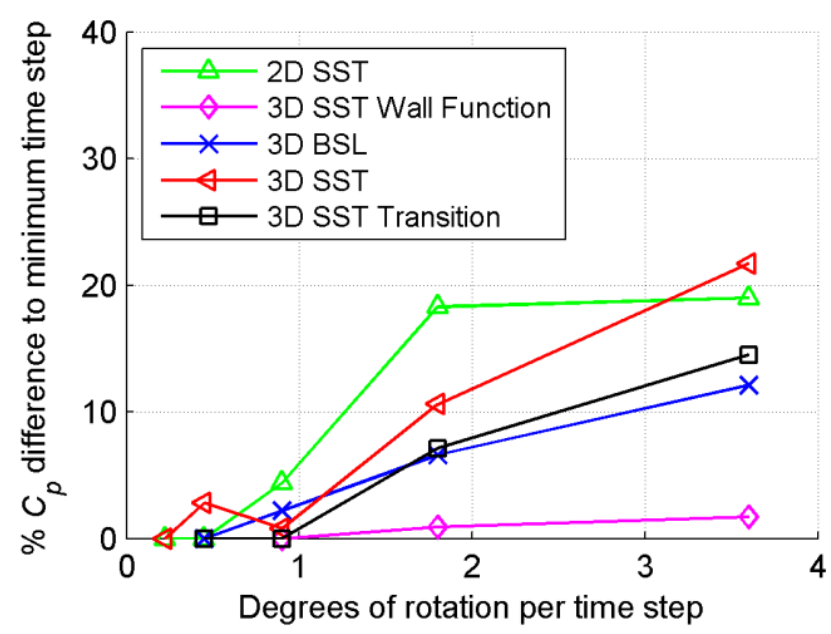

Figure 9: Time Independence for Turbine A CFD models at $\lambda=2.75$ and an inflow velocity of $1.5 \mathrm{~ms}^{-1}$

Figure 10 shows the Turbine A $y+$ independence study, with independence demonstrated at an average $y+<1$ for the 2D, 3D $k-\omega$ SST Wall Function, 3D $k-\omega$ SST, and 3D $k-\omega$ SST Transition models. Independence for the 3D $k-\omega$ SST Wall Function model was demonstrated at $y+=29$. These $y+$ ranges ensured the correct placement of the first mesh cell from the wall for the fully resolved and wall function boundary modeling techniques, and align well with recommended near wall resolution ranges for the turbulence models used [15]. The higher $y+$ of the wall function model resulted in a large reduction in overall mesh element count when compared to the fully resolved model, due to reduction in boundary layer mesh density as shown in Figure 3. Due to dynamic nature of vertical axis turbine hydrodynamic small changes in y+ resulted in small differences in flow field resolution, resulting in small variations in low $y+C_{p}$ as shown in Figure 2.10. However, these fluctuations do not unduly effect simulation results, as the systematic mesh independence studies used ensured than these fluctuations were less than $5 \%$ when compared to the optimal $y+$. The use of reduced $y+$ below $y+=1$ would not significantly increase simulation accuracy, but would unnecessarily increase overall simulation time. The $y+$ range used was also within that suggested by ANSYS [15]. The small increase in $y+$ at values below $y+=1$ is a numerical effect introduced by the small cell sizing at very low $y+$ values.

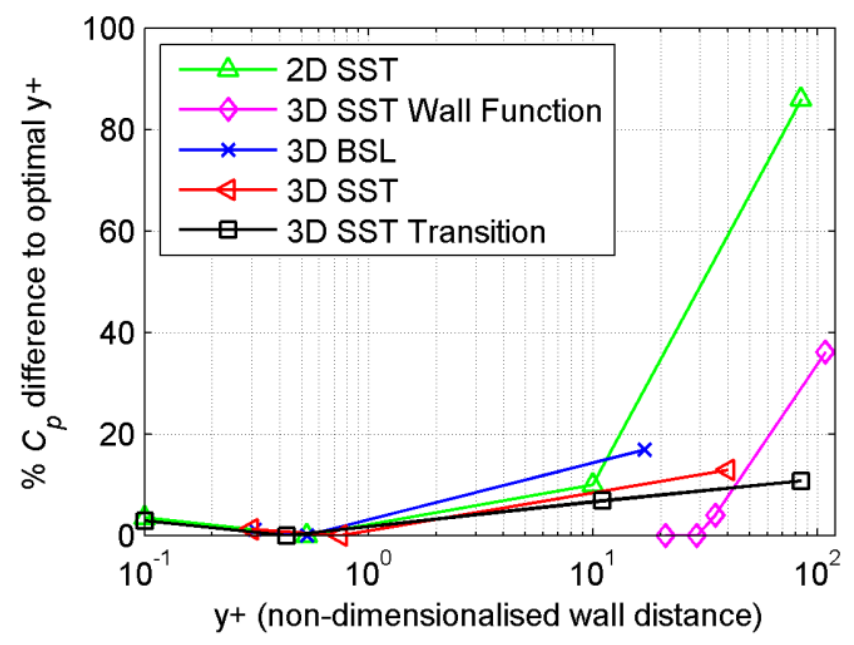

Figure 10: Non-dimensional first cell height $(y+)$ independence study for Turbine $A$ at $\lambda=2.75$ and an inflow velocity of $1.5 \mathrm{~ms}^{-1}$. The optimal $y+$ was approximately $y+=0.75$, except for the 3D $k-\omega$ SST Wall Function model where optimal $y+=29$ 
Domain size studies were performed on Turbine A to ensure spatial solution independence. This was performed by examining the influence of variations of the length, width, and height of the domain on $C_{p}$ whilst keeping all other variables fixed. Simulation results, as shown in Table 3 , indicated that a domain length, width, and height of $20 \mathrm{~d}$, 10s, and 3.33s allowed for domain size independence. Symmetrical independence was confirmed by performing equivalent simulations on the full and half domains as shown in Figure 6 . Total power output differences of less than $0.4 \%$ were demonstrated between the two. This allowed the use of half domains to simulate turbine performance, reducing mesh count and thus total simulation time.

Table 3: Domain Size Independence Study for Turbine A for Half Domain 3D CFD Model Shown in Figure 6 at an Inflow Velocity of $1.5 \mathrm{~ms}^{-1}$ and $\lambda=2.75$

\begin{tabular}{|c|c|c|c|c|c|c|c|c|}
\hline $\begin{array}{l}\text { Domain } \\
\text { Length } \\
\text { (d) }\end{array}$ & $C_{p}$ & $\begin{array}{c}\% C_{p} \\
\text { change } \\
\text { from 20d } \\
\text { case }\end{array}$ & $\begin{array}{l}\text { Domain } \\
\text { Width } \\
\text { (d) }\end{array}$ & $C_{p}$ & $\begin{array}{c}\% C_{p} \\
\text { change } \\
\text { from } 10 d \\
\text { case }\end{array}$ & $\begin{array}{c}\text { Domain } \\
\text { Height } \\
\text { (s) }\end{array}$ & $C_{p}$ & $\begin{array}{c}\% C_{p} \\
\text { change } \\
\text { from } 2.5 s \\
\text { case }\end{array}$ \\
\hline 5 & 0.285 & $11.3 \%$ & 5 & 0.267 & $4.3 \%$ & 1.67 & 0.276 & $7.8 \%$ \\
\hline 20 & 0.256 & - & 10 & 0.256 & - & 3.33 & 0.256 & - \\
\hline 40 & 0.256 & $0.0 \%$ & 20 & 0.251 & $2.0 \%$ & 6.66 & 0.249 & $2.7 \%$ \\
\hline
\end{tabular}

Comparisons were made between low and high order discretisation schemes for Turbine $A$ at $\lambda=2.75$, as shown in Figure 11. The high order scheme resulted in significantly improved accuracy when compared to EFD from literature, with the second order $C_{p}$ results within the reported experimental error shown by the error bars on the EFD results in Figure 11 [16]. This occurred as the second order model was able to capture the highly transient nature of the turbine flow whilst minimizing any numerical diffusion. As a result all simulations were performed using the second order discretisation scheme.

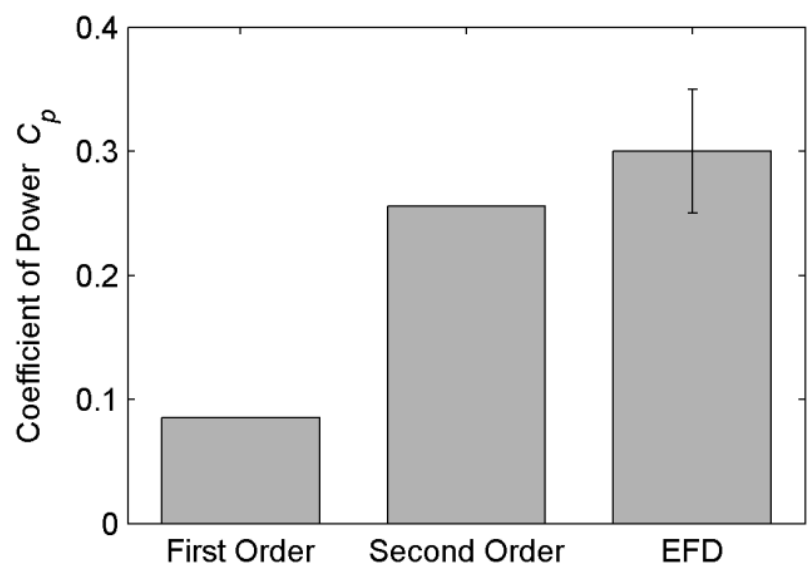

Figure 11: Comparison of first and second order numerical discretisation schemes with EFD $C_{p}$ with error bars from literature for Turbine $A$ at an inflow velocity of $1.5 \mathrm{~ms}^{-1}$ and $\lambda=2.75$ [16]

\section{RESULTS AND DISCUSSION}

Validation of both turbine models was performed against EFD results available in literature for the testing of two geometrically identical turbines conducted at the University of British Columbia's towing tank [16]. Power output was measured using a torque sensor and rotation rate encoder for varying rotational rates from $\lambda=1.5$ to $\lambda=3.5$ and inflow velocities from 1.5 to $2 \mathrm{~ms}^{-1}$. Error bar estimates were only reported for Turbine $A$. 


\subsection{Computational Fluid Dynamics Simulations}

The 2D and 3D CFD and EFD results for the power output characteristics for Turbines $A$ and $B$, at an inflow velocity of $1.5 \mathrm{~ms}^{-1}$, are shown in Figures 12 and 13 respectively.

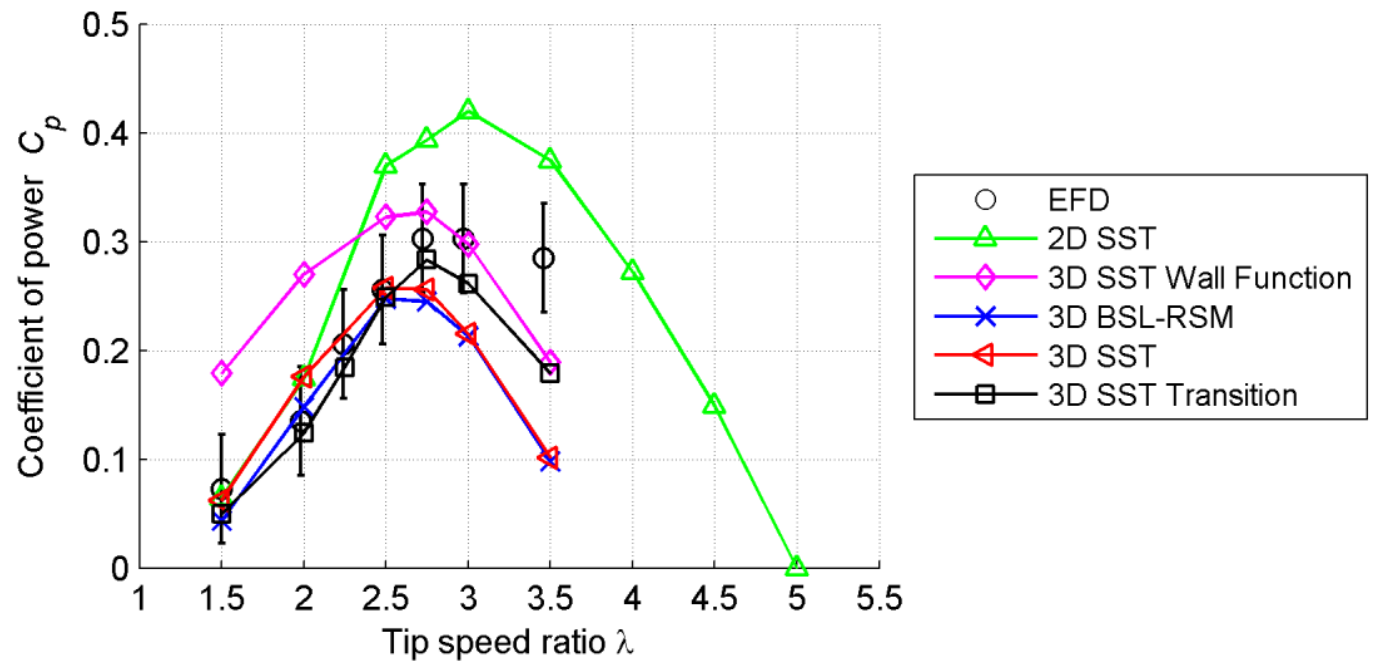

Figure 12: Comparison of $C_{p}-\lambda$ curves for Turbine $A$ at an inflow velocity of $1.5 \mathrm{~ms}^{-1}$.EFD results with error bars from literature [16]

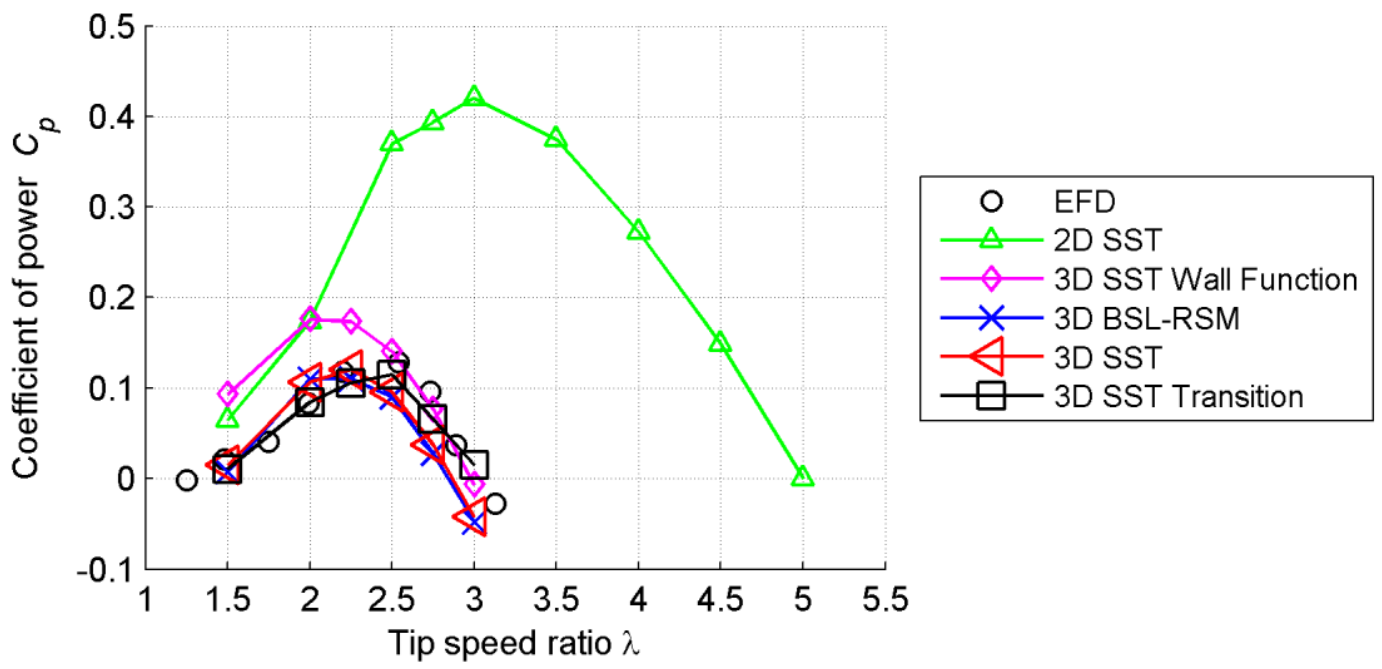

Figure13: Comparison of $C_{p}-\lambda$ curves for Turbine B at an inflow velocity of $1.5 \mathrm{~ms}^{-1}$. EFD results from literature [16]

The 2D k- $\omega$ SST CFD models $C_{p}$ prediction results revealed poor accuracy when compared to the 3D models and EFD from literature [16] as shown in Figures 12 and 13. At $\lambda=1.5$ for Turbine A, $C_{p}$ was predicted to within $11 \%$ of EFD, however predictions of $C_{p}$ for Turbine B were $180 \%$ higher than EFD, as the 2D $k-\omega$ SST model did not account for the significant levels of strut drag on the latter turbine. As $\lambda$ increased $C_{p}$ diverged from the EFD results, with significant prediction errors found at high $\lambda$ for both turbines. These prediction errors occurred as the resistive torque generated by the struts could not be modeled using 2D $k-\omega$ SST CFD models $[2,8]$. These simulation results indicate that 2D $k-\omega$ SST CFD models are unsuitable for the vertical axis turbine simulations, necessitating the use of full 3D simulation models. 
Considering the results from the 3D $k-\omega$ SST Wall Function model against EFD results obtained from literature shown in Figures 12 and 13, at low $\lambda, C_{p}$ was over predicted for both Turbine $A$ and $B$ [16]. Over predictions of $C_{p}$ at $\lambda=1.5$ of $145 \%$ and $304 \%$ were found for Turbines $A$ and $B$ respectively. These occured as the wall function model does not resolve the boundary layer flow down to the sub-viscous layer, but rather applies a generalized log-wall approximation of its shape on the solution. Thus for separated flows, such as at low $\lambda$, the 3D $k-\omega$ SST Wall Function model cannot simulate the high levels of adverse pressure gradients and separation within the boundary layers. As $\lambda$ increased, the $C_{p}$ predictions of the 3D $k-\omega$ SST Wall Function model appeared to slowly converge with the results determined using the fully resolved boundary layer application of the 3D $k-\omega$ SST model, as the increased rotational rate reduces the angle of attack range over the blades. This reduction in angle of attack reduced flow separation and adverse pressure gradients to levels that the 3D $k-\omega$ SST Wall Function model was able to accurately simulate. However, given the poor predictive ability at low to medium $\lambda$, 3D $k-\omega$ SST Wall Function models are a poor choice for vertical axis turbine simulation as they are unable to simulate operational conditions such as start-up and maximum power output accurately.

The highest correlation with EFD for all $\lambda$ was found using the 3D $k-\omega$ SST Transition model, as it accounted for both 3D flow and laminar-to-turbulent flow transition effects. At low $\lambda$ below the $\lambda$ location of maximum $C_{p}$, all $C_{p}$ results were within EFD error bars for Turbine A. For Turbine B at low $\lambda, C_{p}$ predictions were close to EFD, with results for example within $1.3 \%$ of EFD $C_{p}$ at $\lambda=2$. The accuracy of the CFD models resulted from the accurate prediction of flow separation at the high angles of attack experienced at these low $\lambda$ [19]. The transition model also simulated flow transition at low angles of attack that occur at high $\lambda$. The increased $C_{p}$ simulation accuracy when compared to the fully turbulent models is due to better estimation of the wall shear and hence drag on the blades. This results in more accurate prediction of $C_{p}$ at high $\lambda$, as shown in Figure 14.

Using the 3D $k-\omega$ SST Transition model maximum $C_{p}$ was predicted to be within $6.6 \%$ and $10.2 \%$ of EFD results for Turbines $A$ and $B$, as a result of incorporation of all geometric features in the 3D models. Although for Turbine $A$ the $C_{p}$ prediction accuracy at high $\lambda$ above the $\lambda$ location of maximum $C_{p}$ was poor, the shape of the $C_{p}-\lambda$ curve for Turbine $B$ was replicated accurately. This was due to the transition model being able to model the flow transition effects caused by the low average operational Reynolds numbers of approximately 300,000 , as determined using blade chord as the characteristic length.

The effect of flow transition on drag prediction can be seen in Figure 14. Reductions in wall shear stress are shown on the blades and struts when compared to the 3D $k-\omega$ SST fully turbulent solution. Reductions in wall shear and hence drag increase $C_{p}$ at high $\lambda$ as strut resistive torque is reduced. Reductions in wall shear would also improve blade lift to drag efficiency and hence increase $C_{p}$. The poor $C_{p}$ prediction of Turbine $\mathrm{A}$ at high $\lambda$ may be due to experimental inconsistencies at $\lambda=3.5$, as the $C_{p}$ results of Turbine $\mathrm{B}$ at high $\lambda$ were predicted with reasonable accuracy. Over prediction of EFD $C_{p}$ at high $\lambda$ may also occur as a result of blockage effects that were not accounted for in the EFD results, which can artificially increase $C_{p}$ by more than $25 \%$ as shown in previous EFD studies [20]. Prediction errors may also occur due to differences in the turbulence intensity levels between the CFD models and EFD testing, as high turbulence intensity levels can delay stall [21]. This can lead to increases in $C_{p}$ especially at high $\lambda$ [22]. However no turbulence intensity measurements were recorded during EFD testing from literature to compare to CFD turbulence levels. Although the 3D $k-\omega$ SST Transition model demonstrated the highest correlation with EFD results for both turbine models it does have limitations; inherent in its current formulation is the inability to accurately predict cross-flow transition [15]. Although this would not impact significantly on the accuracy of straight-bladed vertical axis turbine simulations, helical turbines may exhibit large degrees of cross-flow separation due to the inclination of the blades to the inflow, which the current ANSYS turbulence model may not accurately capture. 

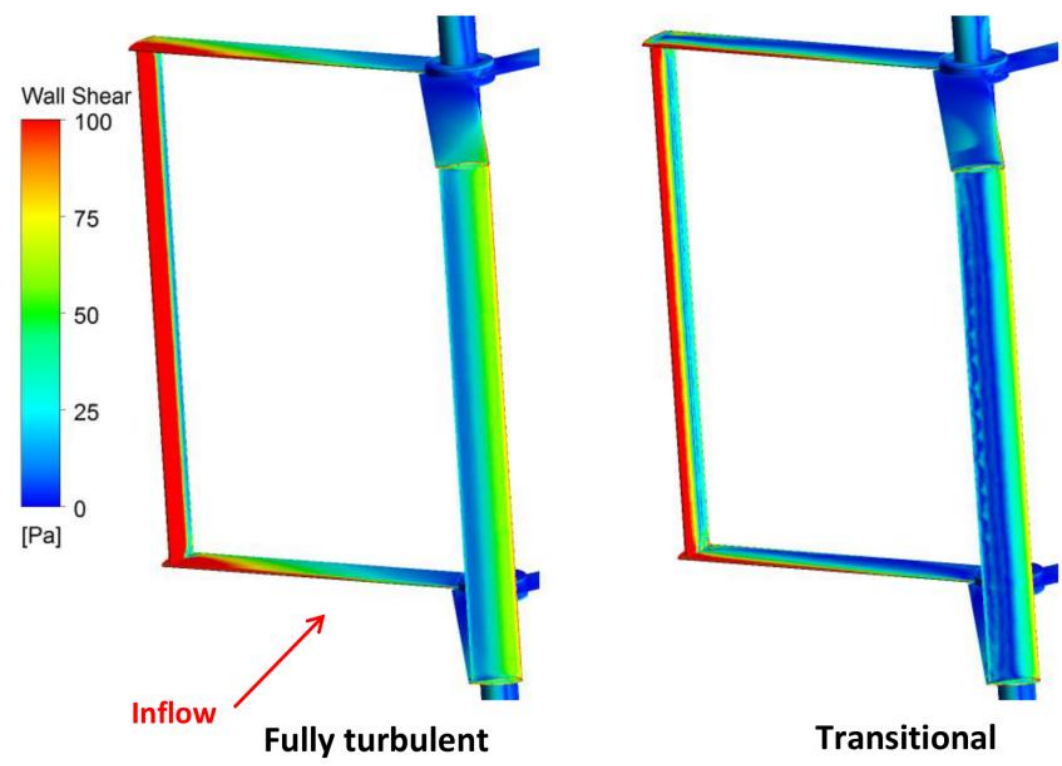

Figure 14: Comparison of the increase in wall shear stress simulated by the 3D $k-\omega$ SST fully turbulent model when compared to the 3D $k-\omega$ SST Transitional models, Turbine $A$ at $\lambda=3.5$ at an inflow velocity of $1.5 \mathrm{~ms}^{-1}$

Although the 3D $k-\omega$ SST Transition model demonstrated the highest correlation with EFD results for both turbine models it does have limitations; inherent in its current formulation is the inability to accurately predict cross-flow transition [15]. Although this would not impact significantly on the accuracy of straight bladed vertical axis turbine simulations, helical turbines may exhibit large degrees of cross-flow separation due to the inclination of the blades to the inflow, which the current ANSYS turbulence model may not accurately capture.

Figures 12 and 13 also show $C_{p}$ simulations of Turbines $A$ and $B$ determined using the 3D $k-\omega$ SST models. At low $\lambda$, good agreement was found between CFD and EFD results for both turbines, with all results being within EFD error bars for Turbine $A$, and close to EFD results for Turbine $B$. Differences between CFD and EFD for Turbine A results were $17 \%$ and $0.8 \%$ at $\lambda=1.5$ and $\lambda=2.5$ respectively, which although high were within reported EFD error bars. Turbine $B$ prediction accuracy at low $\lambda$ was similar, with differences in $C_{p}$ prediction of $14.4 \%$ and $1.7 \%$ at $\lambda=2$ and $\lambda=2.25$ respectively.

Using the 3D $k-\omega$ SST model maximum $C_{p}$ was simulated to within $14.3 \%$ and $6.3 \%$ of EFD results for Turbines $A$ and $B$. This accuracy was a result of the inclusion of all geometry in the 3D models, with Turbine A results falling within reported EFD error bars from literature [16]. However, for both turbines $C_{p}$ prediction accuracy reduced as $\lambda$ increased past the location of maximum $C_{p}$, with the CFD $C_{p}$ values tending to be lower than the equivalent EFD values. The authors suggest that this was due to over prediction of turbine blade and strut airfoil drag due to the use of fully turbulent CFD models, which over estimated skin friction and hence airfoil drag at the low angles of attack experienced at high $\lambda$. This can be seen in Figure 14, where increased levels of wall shear stress were determined by the $k-\omega$ SST model when compared to the $k$ - $\omega$ SST Transition model predictions.

The 3D BSL-RSM model provided reduced $C_{p}$ prediction accuracy when compared to the 3D $k-\omega$ SST models for all $\lambda$ as shown in Figures 12 and 13. Below $\lambda=3$, all results from Turbine $A$ were within the EFD error bars, however $C_{p}$ differences between the CFD and EFD results of $40.4 \%$ and $9.6 \%$ at $\lambda=1.5$ and $\lambda=2$ respectively were higher than those of the $k-\omega$ SST models. The prediction accuracy for Turbine B at low $\lambda$ was also poor, with differences in $C_{p}$ prediction being $64 \%$ and $32 \%$ 
at $\lambda=1.5$ and $\lambda=2$ respectively. Maximum $C_{p}$ was predicted to within $18.2 \%$ and $14.1 \%$ of the EFD results for Turbines $A$ and $B$ respectively, notably with less accuracy than the 3D $k-\omega$ SST model. It is difficult to ascribe reasons as to this reduction in accuracy when compared to the $k-\omega$ SST models, however it appear that the BSL-RSM may be simulating separation at a lower angle of attack than the $k-\omega$ SST models. The prediction accuracy of the BSL-RSM model reduced at high $\lambda$, which the authors ascibed to experimental inconsistencies, blockage effects, and transitional effects similar to that noted for the $k-\omega$ SST model simulations. Solutions using the BSL-RSM model, with its additional transport and dissipation equations, did not improve simulation accuracy when compared to the standard isotropic eddy-viscosity based models.

\subsection{Computational Requirements and Numerical Simulation Recommendations}

The computational efficiency of the turbulence model and 2D and 3D investigations were established by comparing simulation time and computer cluster core requirements to simulate one revolution as shown in Table 4 at $\lambda=2.75$. All simulations were performed on a distributed cluster comprising of Intel Xeon 5160 3.0GHz processors with 2GB memory per core.

Table 4: Simulation Time and Computational Requirements for One Turbine Revolution. Turbine A at $\lambda=2.75$ at an Inflow Velocity of $1.5 \mathrm{~ms}^{-1}$.

\begin{tabular}{cccccc}
\hline $\begin{array}{c}\text { Turbulence } \\
\text { Model }\end{array}$ & 2D $\boldsymbol{k}-\boldsymbol{\omega}$ & 3D $\boldsymbol{k}$ - $\boldsymbol{\omega}$ SST & 3D & 3D $\boldsymbol{k}-\boldsymbol{\omega}$ & 3D $\boldsymbol{k}$ - $\boldsymbol{\omega}$ SST \\
SST & Wall Function & BSLRSM & SST & Transition \\
\hline Time (minutes) & 90 & 400 & 2700 & 1200 & 6200 \\
Cores & 4 & 16 & 24 & 24 & 24 \\
\hline
\end{tabular}

\subsection{Numerical Simulation Recommendations}

Key conclusions were gained from evaluations of power output prediction accuracy when combined with the determination of computational resource requirements for each model, namely that:

- 2D k- $\omega$ SST models were computationally efficient due to reduction in mesh size when compared to the 3D models. However $C_{p}$ prediction accuracy was very poor when compared to EFD, as strut and end influences were not simulated;

- 3D k- $\omega$ SST Wall Function models offered reduced simulation times than the fully resolved models, as a result of significant reductions in mesh size. However $C_{p}$ prediction accuracy was poor when compared to the EFD, as it were unable to model separation at low rotational rates accurately;

- 3D BSL-RSM models were computationally demanding due to the extra seven transport and dissipation equations solved. However $C_{p}$ prediction accuracy was reduced when compared to 3D $k-\omega$ SST models and EFD due to possible separation prediction error;

- 3D $k-\omega$ SST Transition models were accurate but required excessive computation times due to large mesh element counts necessary for element count independence, as well as for the solution of the additional transition equations. Overall simulation time increased by a factor of more than five when compared to the 3D $k$ - $\omega$ SST models;

- A suitable balance between computational requirements and power output prediction accuracy was found using turbulent 3D $k-\omega$ SST models.

\subsection{Geometrical Effects on Power Output}

The influences of geometrical design on power output were captured by the 3D CFD models as shown in Figures 12 and 13. Using the 3D $k$ - $\omega$ SST model, Turbine A power output was found to 
be more than double that of Turbine $B$, as although the turbines shared the same blade section, they differed in strut section location, cross-section and mounting tab design. This result is consistent with the $136 \%$ increase in power output efficiency found by EFD from literature [16]. The use of 3D CFD models allows differences of power output caused by geometrical design to be quantified without the need for EFD. If investigation of the relative performance of blade section variations is desired it may be possible to use 2D CFD models, however they will poorly capture total power output and hence should not be used for determining overall power generation capacity.

\subsection{Flow Visualisation}

The use of CFD also allows for flow visualisation without the expense and difficulty of EFD methods such as Particle Image Velocimetry (PIV) [23]. Visualisation of vortex shedding for the 2D and 3D $k-\omega$ SST models is shown in Figures 15 and 16. The 2D $k-\omega$ SST models were unable to capture any strut or blade tip losses due to the geometrical layout of vertical axis turbines, and can only simulate blade and shaft vortex shedding. As a result the 2D $k-\omega$ SST poorly predicts $C_{p}$ as shown in Figures 12 and 13. Conversely, the 3D $k-\omega$ SST CFD models resolved all key hydrodynamic flow features including blade tip and strut vortex shedding as shown in Figure 16, due to the inclusion of all geometrical features. This inclusion results in increased simulation accuracy when compared to the 2D $k-\omega$ SST model, as shown in Figures 12 and 13. Using CFD models differential analysis of vortex shedding between turbines of differing geometrical layouts is also possible [13]. Validation of the vortex shedding shown in Figures 15 and 16 was not possible as the EFD results included no flow visualisation, although validation could be performed using EFD techniques such as Particle Image Velocimetry (PIV). These comparisons would also allow for the evaluation of the influence of flow diffusion effects caused by the increase in mesh size away from the turbine surfaces on vortex shedding simulation accuracy.

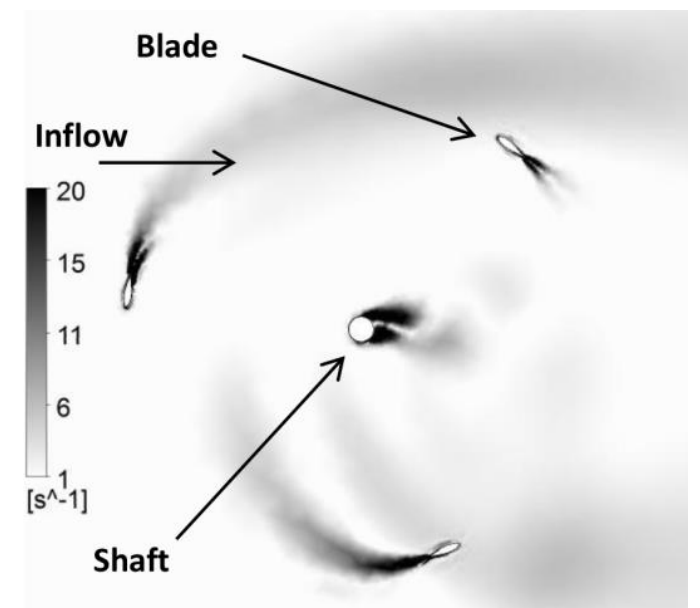

Figure 15: Vortex structure visualisation for Turbine A at $\lambda=2.75$ for the 2D $k-\omega$ SST model. Vorticity in stationary frame from 1 to $20 \mathrm{~s}^{-1}$ at an inflow velocity of $1.5 \mathrm{~ms}^{-1}$ 


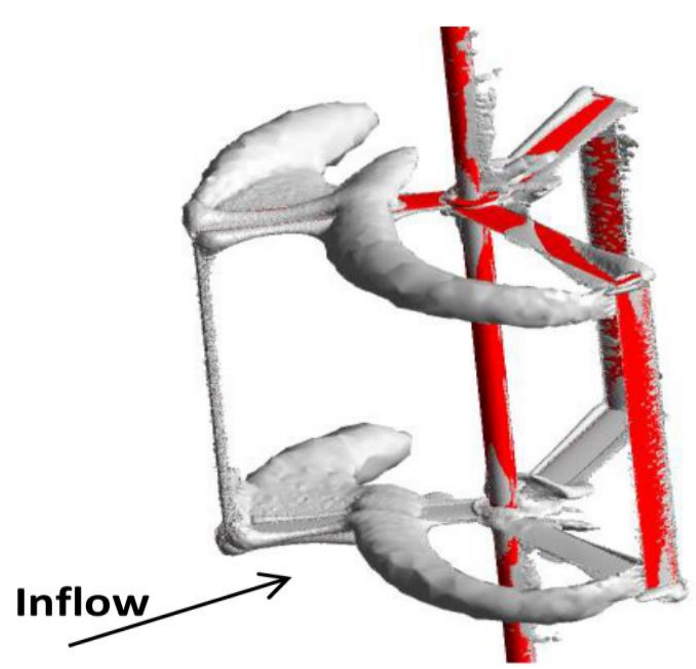

Figure 16: Vortex shedding visualisation for Turbine $A$ at $\lambda=2.75$ for the 3D $k-\omega$ SST model. Vorticity of $16 \mathrm{~ms}^{-1}$ at an inflow velocity of $1.5 \mathrm{~ms}^{-1}$ at $\lambda=2.75$.

\section{CONCLUSIONS}

The power output of two vertical axis turbines was simulated using 2D and 3D CFD models with varying turbulence and boundary layer flow modeling techniques. Although the $k-\omega$ SST Transition model resulted in the highest correlation with experimental power output results, the authors suggest that the 3D $k-\omega$ SST model is better suited for vertical axis turbine simulation. It offers comparable accuracy to the $k-\omega$ SST Transition model at low to medium rotational rates, as well as similar accuracy for maximum power output predictions, without the additional meshing and excessive computational expense of the transition model.

The development of CFD flow transition models is ongoing, which will hopefully reduce the computational requirements of transitional models which were found to be excessive in this study. The authors suggest that this study be revisited as transition models improve, as they show promising results especially at high rotational rates.

\section{NOMENCLATURE}

$C_{m} \quad$ Moment Coefficient

$C_{p} \quad$ Power Coefficient

$d \quad$ Turbine diameter $(\mathrm{m})$

$r \quad$ Radius $(m)$

$s \quad$ Blade span $(\mathrm{m})$

$S \quad$ Turbine Frontal Area $\left(\mathrm{m}^{3}\right)$
$V \quad$ Inflow Velocity $\left(\mathrm{ms}^{-1}\right)$

$y+\quad$ Non-dimenional first cell wall distance

$\lambda \quad$ Tip Speed Ratio

$\rho \quad$ Density $\left\{\mathrm{kgm}^{-3}\right)$

$\omega$ Rotational Rate $\left(\right.$ rads $\left.^{-1}\right)$

\section{REFERENCES}

[1] IPCC. IPCC Special Report on Renewable Energy Sources and Climate Change Mitigation. Prepared by Working Group III of the Intergovernmental Panel on Climate Change [Edenhofer O, Pichs-Madruga R, Sokona Y, Seyboth K, Matschoss P, Kadner S, Zwickel T, Eickemeier P, Hansen G, Schlömer S, von Stechow C(eds)]. Cambridge University Press, Cambridge, United Kingdom and New York, NY, USA. 2011. 
[2] Marsh P, Ranmuthugala D, Penesis I, Thomas G. Performance predictions of a straight-bladed vertical axis turbine using double-multiple streamtube and computational fluid dynamics. The Journal of Ocean Technology, 8(1), 2013, pp. 87-103.

[3] Scheurich F, Fletcher T, Brown, R. Simulating the aerodynamic performance and wake dynamics of a vertical-axis wind turbine. Wind Energy, 14(2), 2010, pp. 159-177.

[4] Castelli MR, Ardizzon G, Battisti L, Benini E, Pavesi G. Modeling strategy and numerical validation for a Darrieus vertical axis micro-wind turbine. ASME 2010 International Mechanical Engineering Congress and Exposition, 2010. Vancouver, British Columbia, Canada, pp. 409-418.

[5] Malipeddi AR, Chatterjee D. Influence of duct geometry on the performance of Darrieus hydroturbine. Renewable Energy, 43, 2012, pp. 292-300.

[6] Danao, LJ, Edwards, J, Eboibi, O, Howell, R. The performance of a vertical axis wind turbine in fluctuating wind - a numerical study. Proceedings of the World Congress on Engineering, 3-5 July 2013, 2013, London, U.K.

[7] Maitre T, Amet E, Pellone C. Modeling of the flow in a Darrieus water turbine: Wall grid refinement analysis and comparison with experiments. Renewable Energy, 51, pp. 497-512.

[8] Marsh P, Ranmuthugala D, Penesis I, Thomas G., Three dimensional numerical simulations of a straight-bladed vertical axis tidal turbine, Proceedings of the 18th Australasian Fluid Mechanics Conference, 3-7 December 2012, Launceston, Tasmania, Australia.

[9] Dai YM, Lam W. Numerical Study of Straight-Bladed Darrieus-Type Tidal Turbine. Energy, 162, 2009, pp. 67-76.

[10] Nobile R, Vahdati M, Barlow J, Mewburn-Crook A. Dynamic stall for a Vertical Axis Wind Turbine in a two-dimensional study. In: World Renewable Energy Congress 2011, Sweden.

[11] Lain S. Simulation and Evaluation of a Straight-Bladed Darrieus-type Cross Flow Marine Turbine. Journal of Scientific and Industrial Research, 69, 2010, pp. 906-912.

[12] Gretton GI. The hydrodynamic analysis of a vertical axis tidal turbine. PhD dissertation, University of Edinburgh, 2009.

[13] Marsh P, Ranmuthugala D, Penesis I, Thomas G., Three-dimensional numerical simulations of straight-bladed vertical axis tidal turbines investigating power output, torque ripple and mounting forces. Renewable Energy, 83, pp. 67-77.

[14] Marsh P, Ranmuthugala D, Penesis I, Thomas G., Numerical investigation of the influence of blade helicity on the performance characteristics of vertical axis tidal turbines. Renewable Energy 81, 2015, pp. 926-935.

[15] Ansys Inc. ANSYS CFX-Solver Theory Guide: Release 13.0, 2010. Canonsburg, Pennsylvania.

[16] Rawlings G. Parametric Characterization of an Experimental Vertical Axis Hydro Turbine. MSC dissertation. University of British Columbia, 2008.

[17] Menter FR. Two-Equation Eddy-Viscosity Turbulence Models for Engineering Applications. AIAA J, 32(8), 1994, pp. 598-1605. 
[18] J.D. Anderson, Fundamentals of Aerodynamics, McGraw-Hill Education, 2010

[19] Paraschivoiu I. Wind Turbine Design: With Emphasis on Darrieus Concept. Quebec, Canada: Polytechnic International Press, 2002.

[20] Bachant P, Wosnik M. Performance measurements of cylindrical- and spherical-helical cross-flow marine hydrokinetic turbines, with estimates of exergy efficiency. Renewable Energy, 74, 2015, pp. 318-325.

[21] Leu TS, Yu JM, Hu CC, Miau JJ, Liang SY, Li JY, Cheng JC, Chen SJ. Experimental Study of Free Stream Turbulence Effects on Dynamic Stall of Pitching Airfoil by using Particle Image Velocimetry. Applied Mechanics and Materials, 225, 2012, pp. 103-108.

[22] Maganga F, Germain G, King J, Pinon G, Rivoalen E. Experimental characterisation of flow effects on marine current turbine behaviour and on its wake properties. IET Renewable Power Generation, 4(6), 2010, pp. 498-509.

[23] Ferreira, C.J. The near wake of a VWAT: 2D and 3D views of the VWAT aerodynamics. PhD dissertation. Delft University of Technology, 2009. 